\title{
Proteomics: a new era in pediatric acute myeloid leukemia
}

\section{research}

\author{
Jatinder K. Lamba' and Stanley Pounds ${ }^{2,3}$ \\ ${ }^{1}$ Pharmacotherapy and Translational Research, Center for Pharmacogenomics and Personalized Medicine, UF Health Cancer Center, \\ University of Florida, Gainesville, FL, ${ }^{2}$ Biostatistics, St. Jude Children's Research Hospital, Memphis, TN and ${ }^{3}$ Oncology, St. Jude Children's \\ Research Hospital, Memphis, TN, USA \\ E-mail: jlamba@cop.ufl.edu \\ https://doi.org/10.3324/haematol.2021.280305
}

In this issue of Haematologica, Hoff et al. ${ }^{1}$ report their findings on the proteome of 500 pediatric cases of acute myeloid leukemia (AML) and 30 control $\mathrm{CD} 34^{+}$samples. Over the past few years, it has become clear that proteomic evaluations are critical to fully understand tumor biology and develop promising therapies in oncology. As recently reviewed by Kwon et al., ${ }^{2}$ several studies in solid tumors have led to identification of druggable protein targets as well as protein biomarkers of prognostic relevance. Largescale efforts such as the National Cancer Institute's Clinical Proteomics Tumor Analysis Consortium (CPTAC) program have successfully identified relevant signatures for multiple cancers (https://proteomics.cancer.gov/programs/cptac). Despite these efforts, proteomic profiling of hematologic malignancies has been sparse and limited, especially in pediatric AML. One study evaluated a few proteins by twodimensional gel electrophoresis and matrix assisted laser desorption ionization - time of flight (MALDI-TOF) analysis in three pediatric AML patients; ${ }^{3}$ another study compared 31 proteins between 16 pediatric AML patients and five controls ${ }^{4}$ and we recently evaluated global proteomic profiles of 16 pediatric AML patients. ${ }^{5}$ We applaud Hoff et al. ${ }^{1}$ for collecting, analyzing, and sharing their data from a very large cohort of patients treated uniformly under a large cooperative group clinical trial protocol. The pediatric AML research community will gain valuable insights from these results and data for decades to come.

The article reports the results of profiling 296 candidate proteins by reverse phase protein arrays, a technology that this team has previously shown to produce reliable data that are robust against technical pre-analytical factors such as shipping, transit time, and temperature. ${ }^{6}$ Using their own MetaGalaxy (https://www.leukemiaatlas.org/code) and progenyClust methods ${ }^{7}$ the authors compressed these 296 individual protein variables into 31 biologically annotated protein function groups and then 12 protein constellations to eventually assign each patient to one of nine protein signature classes. Intriguingly, their downstream analyses found that patients with one protein signature class had better outcomes with Ara-C (cytarabine), daunorubicin, and etoposide (ADE) plus bortezimib (ADEB) than with $A D E$ alone and that patients with another protein signature class had better outcomes with ADE than with ADEB. This raises the tantalizing hope that it may be possible to use proteomic evaluations to develop personalized therapy and that patients with some protein signature classes may also have reasonable targeted drug options available to them.

The study additionally showed that this kind of clinically vital information is unlikely to be available from RNA transcriptomic profiling. In this study, RNA expression levels typically showed a fairly weak correlation with protein expression levels (median Pearson correlation $=0.17$ ). We obtained similar results in our pilot global proteomic study. ${ }^{5}$ These findings provide the motivation to systematically evaluate the global proteome in large clinical trial cohorts to uncover valuable biological and clinical insights regarding the impact of other proteins in pediatric AML.

The paper by Hoff et al. ${ }^{1}$ thus marks the dawn of a new era in pediatric AML multi-omics research, instigating researchers to evaluate the global pediatric AML proteome and integrate its key components with relevant elements of other-omics profiles, such as the transcriptomic leukemia stem cell scores ( $\mathrm{LSC}_{17},{ }^{8}$ and $\mathrm{pLSC}^{\circ}{ }^{\circ}$ ), prognostic transcriptomic subgroups ${ }^{10}$ and the pediatric AML methylome. ${ }^{11}$ In this new era it will be imperative to develop and apply scientifically innovative and statistically rigorous methods of data analysis in order to obtain clinically and biologically useful insights based on widely reproducible results. We have incorporated subject-level bootstrap resampling of entire intact molecular profiles ${ }^{12-}$

${ }^{14}$ into our own recent work because these methods can help to quantify the reproducibility of the results of complex multistage data analysis algorithms. We look forward to exploring how to incorporate annotation-informed data reduction schemes, such as the one used in MetaGalaxy, into the subject-level bootstrap resampling framework of well-established statistical rigor.

Advances in the field of mass spectrometry in the last couple of decades have enabled high-throughput collection of global proteomic profiles. Integrating these profiles with genomics and transcriptomics can enhance our ability to perform systems-biology-based investigations to accelerate clinical translation by defining disease heterogeneity, establishing biomarkers predictive of response/relapse and last but not least identify promising novel drug targets. Although clinical translation of research discoveries is challenging, there are successful 
examples such as various Food and Drug Administration (FDA)-cleared/approved proteomic biomarkers currently in clinical use (predominantly immunohistochemistry or immunoassay-based testing of PSA, AFP, Her-2/neu, PR, $E R, c-k i t$, etc.) for several solid malignancies. The recent introduction of FDA-approved biomarkers (such as PD-L1) has enhanced the personalized use of immune checkpoint inhibitors. The advances in other malignancies should stimulated the expansion of these strategies to pediatric cancer and, specifically, to hematologic malignancies such as AML. As also reflected by the authors, longitudinal unbiased global proteomic studies in bulk tissue and single cells hold promise for identifying relapse/resistant clones and specifically targeting them using novel agents. Future studies are required not only to establish and validate potential biomarkers in large, independent cohorts of patients but also to develop diagnostic tests with rapid turnaround to enhance the clinical translation of proteomics into treatment decision-making.

\section{Disclosures}

No conflicts of interest to disclose.

\section{Contributions}

$J K L$ and SP wrote the editorial.

\section{Funding}

JKL and SP are supported by NIH R01CA132946-12A1.

\section{References}

1. Hoff FW van Dijk AD, Qiu Y, et al. Clinical relevance of proteomic profiling in de novo pediatric acute myeloid leukemia: a Children's Oncology Group study. Haematologica. 2022;107(10):2329-2343

2. Kwon Y-W, Jo HS, Bae S, et al. Application of proteomics in cancer: recent trends and approaches for biomarkers discovery. Front Med (Lausanne). 2021;8:747333.

3. Braoudaki M, Tzortzatou-Stathopoulou F, Anagnostopoulos AK, et al. Proteomic analysis of childhood de novo acute myeloid leukemia and myelodysplastic syndrome/AML: correlation to molecular and cytogenetic analyses. Amino Acids. 2011;40(3):943-951.

4. Levine JH, Simonds EF, Bendall SC, et al. Data-driven phenotypic dissection of AML reveals progenitor-like cells that correlate with prognosis. Cell. 2015;162(1):184-197.

5. Nguyen NHK, Wu H, Tan H, et al. Global proteomic profiling of pediatric AML: a pilot study. Cancers (Basel). 2021;13(13):3161.

6. Horton TM, Hoff FW, van Dijk A, et al. The effects of sample handling on proteomics assessed by reverse phase protein arrays (RPPA): functional proteomic profiling in leukemia. $J$ Proteomics. 2021;233:104046.

7. Hu CW, Qutub AA. progenyClust: an R package for progeny clustering. The R Journal. 2016;8:10.

8. Ng SW, Mitchell A, Kennedy JA, et al. A 17-gene stemness score for rapid determination of risk in acute leukaemia. Nature. 2016;540(7633):433-437.

9. Elsayed AH, Rafiee R, Cao X, et al. A six-gene leukemic stem cell score identifies high risk pediatric acute myeloid leukemia. Leukemia. 2020;34(3):735-745.

10. Fornerod M, Ma J, Noort S, et al. Integrative genomic analysis of pediatric myeloid-related acute leukemias identifies novel subtypes and prognostic indicators. Blood Cancer Discov. 2021;2(6):586-599.

11. Lamba JK, Cao X, Raimondi SC, et al. Integrated epigenetic and genetic analysis identifies markers of prognostic significance in pediatric acute myeloid leukemia. Oncotarget. 2018;9(42):26711-26723.

12. Efron B. Bootstrap methods: another look at the jackknife. Annals Stat. 1979;7:26.

13. Dudoit S, Fridlyand J. A prediction-based resampling method for estimating the number of clusters in a dataset. Genome Biol. 2002;3(7):RESEARCH0036.

14. Dudoit S, Fridlyand J. Bagging to improve the accuracy of a clustering procedure. Bioinformatics. 2003;19(9):1090-1099. 